Mögliche Systemdynamiken eines Handels mit Emissionsrechten

\title{
Kioto und die Folgen
}

\section{Ein Ergebnis der Kioto-Konferenz ist die Eröffnung der Möglichkeit eines Emissionshandels. Gerade dies stößt bei Umweltschutzorganisationen jedoch auf Ablehnung. Die Wirkungen einer solchen Regulierung hängen allerdings entscheidend vom Design des Handelssystems ab.}

\section{$\mathrm{D}$} Von Wilhelm Althammer verbindlich vorgesehenen Reduktionsziele für Treibhausgase gelten für die westlichen Industrienationen und die osteuropäischen Transformationsländer. Sie sind im Zeitraum 2008-2012 zu erfïllen und betragen im Durchschnitt etwa 5 Prozent, wobei als Basisjahr 1990 (für Kohlendioxid, Methan und Lachgas) bzw. 1995 (für perfluorierte Kohlenwasserstoffe u.a.) dient.

Von Kritikern werden die beschlossenen Reduktionen als zu gering beurteilt - beispielsweise erachtete der Wissenschaftliche Beirat Globale Umweltveränderungen eine 23-prozentige Reduktion bis 2010 für notwendig (1). Daneben wird auch die ungleiche Verteilung der Reduktionen kritisiert: Die Vorgaben reichen von Reduktionen (u.a. EU um 8, USA um 7, Japan um 6 Prozent) über Stabilisierungen (u.a. Rußland) bis hin zur Möglichkeit der Erhöhung der Emissionen (u.a. Australien um 8 Prozent). Kritisiert wurde auch die vorgesehene Möglichkeit eines Handels mit Emissionen. Das Hauptproblem wird darin gesehen, daß in Rußland die aktuellen Emissionen aufgrund des wirtschaftlichen Zusammenbruchs um etwa 30 Prozent unter dem Zielwert für 2012 liegen. Wenn dann die USA einen Teil ihrer Reduktionsverpflichtung in Rußland 'einkauft', resultiert ein 'hot air trading', da Emissionen, die bei realistischer Sichtweise gar nicht angefallen wären, zur Erfüllung der Vorgaben dienen.

Der häufig gebrauchte Begriff des 'Ablaßhandels' weckt Assoziationen an Schuld (der Industrienationen am Treibhauseffekt) und Sühne, die "politisch korrekt" nur durch eigene Emissionsreduktionen geleistet werden kann. Diese moralische Sichtweise mag zwar populär sein, sie verstellt jedoch den Blick auf die Tatsache, daß Erfolge beim Klimaschutz eine kostenminimierende Umsetzung der Reduktionsziele voraussetzen - nur dann ist eine effektive Politik und die Partizipation einer großen Zahl von Ländern möglich. Die Aufgabe besteht deshalb darin, das Handelssystem entsprechend auszugestalten. Das Kioto-Protokoll läßt jedoch das Designproblem offen und verweist hierzu auf die Folgekonferenzen. Für den Erfolg des Protokolls ist das Systemdesign jedoch essentiell: So machte die deutsche Umweltministerin die Ratifizierung des Protokolls davon abhängig, daß mindestens 50 Prozent der Reduktionsverpflichtung durch nationale Maßnahmen erbracht werden.

\section{- Probleme des Systemdesigns}

Welche Elemente muß die Implementierung eines idealen Handelssystems (2) umfassen? Treibhausgasemissionen weisen zwei Eigenschaften auf, die für einen globalen Handel sprechen: Zum einen unterscheiden sich die Vermeidungskosten international, was ein Potential für Kostenreduktionen eröffnet. Zum anderen spielt der Ort der Emission für den Effekt auf die globale Erwärmung keine Rolle - durch Handel können Reduktionen deshalb dort vorgenommen werden, wo sie die geringsten Kosten verursachen, ohne daß lokale 'hot spots' zu befürchten wären.

Handelssysteme: Hier gibt es zwei grundlegende Möglichkeiten. Das 'cap and trade'-System beruht auf einem expliziten Markt für ungenutzte Emissionsrechte, die vorher für jedes Land verbindlich festgelegt wurden. Beim 'baseline and credit'-System erwirbt ein Land handelbare Emissionsgutschriften, wenn es unter den durch ein Basisszenario bestimmten zulässigen Gesamtemissionen bleibt. Konkrete Erfahrungen liegen mit beiden Handelssystemen in den USA vor (3). Die gemeinsame Finanzierung von Vermeidungsmaßnahmen (joint implementation) stellt einen Spezialfall dar, bei dem Reduktionen gegenuiber einem Basisszenario dem ausländischen Investor zwar angerechnet werden. Allerdings ist der dafür entrichtete Preis projektgebunden und steht dem Empfängerland nicht zur freien Verfiigung. Diese sogenannte 'joint implementation', die schon in der Klimarahmenkonvention von 1992 explizite Erwähnung fand, befindet sich noch in einer Testphase - anrechenbare Gutschriften können derzeit noch nicht erworben werden.

Allokation der Emissionsrechte: Handel setzt die Existenz rechtlich geschützter Eigentumstitel und damit die Einigung auf verbindliche Emissionsgrenzen voraus. Dieser erste Schritt ist mit gravierenden distributiven Konsequenzen verbunden: Die Verteilung der Grenzen determiniert die Richtung der späteren Zahlungsströme und damit die Nettoauszahlung aus einer Teilnahme an einem Klimaabkommen.

Zwei extreme Möglichkeiten, nämlich Allokation nach einem historischen Schlïssel und nach Bevölkerungsgröße, zeigen dabei die Bandbreite möglicher Interessenkonflikte.

Eine Verteilung nach historischen Emissionen in einem Basisjahr wäre für die Industrienationen am vorteilhaftesten, da sie die aktuellen Produktionsstrukturen wiedergibt und $\mathrm{so} \mathrm{zu}$ relativ geringen Anpassungskosten führt. Allerdings sprechen sowohl Gerechtigkeits-aspekte (Verursacherprinzip) als auch die Tatsache, daß sich die regionale Verteilung der Emissionen in den nächsten Dekaden ändern wird, dafür, daß Entwicklungsländer eine derartige Allokation ablehnen. Da nämlich ihre Kohlendioxid-Emissionen erheblich steigen werden, würde man ihre Wachstumsmöglichkeiten beschränken, wenn die Ausstattung an Rechten ihren Emissionsanteil der 90er-Jahre wiedergibt.

Hinter einer Verteilung nach der Bevölkerungsgröße steht die einfache egalitäre Gerechtigkeitsvorstellung, daß jeder Mensch ein gleiches Recht auf die Nutzung von Ressourcen in globalem Gemeineigentum hat. Ihre Realisierung dürte an den ökonomischen Konsequenzen scheitern: So profitierten zwar bevölkerungsreiche Länder mit niedrigem Volkseinkommen, OECD-Länder und osteuropäische Länder hätten dagegen die höchsten Belastungen, weshalb sie kaum einer derartigen Lösung zustimmen würden.

Ein pragmatischer Mittelweg für die Verteilung ist bis jetzt noch nicht gefunden worden. Allerdings muß dieses Problem bei jedem Klimaabkommen gelöst werden, unabhängig davon, ob ein Handel stattfindet. Dieser würde die Lösung erleichtern, da insbesondere Entwicklungsländer eine Einnahmequelle und somit Anreize zur Partizipation an einem globalen Abkommen erhielten. 
Handelspartner: Als Handelspartner kommen primär Staaten in Frage, die einer verbindlichen Reduktionsverpflichtung unterliegen. Die nationale Politik für die Umsetzung der Vorgaben würde durch den Handel nur dadurch beschränkt, daß sie kompatibel zu ihm sein muß. Eine Politik, die alleine auf Emissionsabgaben setzt, wäre weniger geeignet, da diese bei Unsicherheit über die Reaktionen der Belasteten ökologisch wenig treffsicher sind. Eine sichere Planung des jährlichen Bedarfs an Emissionsrechten wäre somit ausgeschlossen.

Alternativ könnten auch auf nationaler Ebene Emissionsrechte ausgegeben werden. Dies ist allerdings für die rund 50\% Kleinemittenten aufgrund der Transaktionskosten problematisch. Wenn aber zumindest die Großemittenten von Treibhausgasen (wie Kraftwerke) mit Emissionsrechten ausgestattet werden, spricht nichts dagegen, sie auch am internationalen Handel zu beteiligen. Dies würde die Marktbreite erhöhen und Probleme der Marktmacht reduzieren.

Fraglich ist, inwieweit auch Länder ohne verbindliche Reduktionsziele am Handel teilnehmen können. Das Kyoto-Protokoll sieht dies zwar vor, da diese Länder jedoch keine Rechtstitel besitzen, käme höchsten ein Handel auf der Basis von konkreten Projekten in Frage. Dieser dürfte allerdings mit hohen Transaktionskosten verbunden sein: So müssen Handelspartner auf bilateraler Ebene gefunden sowie verifizierbare Basisund Reduktionsszenarien entwickelt werden. $\mathrm{Da}$ keine völkerrechtliche Bindung an das Basisszenario besteht, läge das Risiko der Nichterfiillung der Reduktion und mithin des nachträglichen Verfalls der Gutschrift alleine beim Erwerber.

Daneben bestehen eher technische Probleme bei der Definition und Standardisierung des Handelsguts (Kohlendioxid-Äquivalente versus eigene Märkte für jedes Gas) sowie der Auswahl und Kontrolle der Handelsintermediäre (z.B. die existierenden Rohstoffbörsen).

Überwachung und Durchsetzung: Damit der internationale Handel funktioniert, müssen die Marktteilnehmer Vertrauen in das Handelssystem haben. Vertrauen basiert auf Information und damit auf der Fähigkeit, Treibhausgasemissionen auf nationaler Ebene korrekt zu erfassen und nachvollziehbar $\mathrm{zu}$ dokumentieren. Eine internationale Behörde hätte dann die Aufgabe, die nationalen Rechnungslegungen zu kontrollieren und Sanktionen bei Vertragsverletzungen zu veranlassen. Diese Behörde funktioniert wie

\begin{tabular}{|c|c|c|}
\hline A & 0 Land X & p \\
\hline $\begin{array}{l}\text { Erstausstattung on ER } \\
\text { Reserve an ER } \\
\text { Zukäufe von ER } \\
\text { Schaffung von Senken } \\
\text { Zerstörung von THGS }\end{array}$ & $\begin{array}{l}\text { Tatsächliche Emissionen } \\
\text { Verkäute von ER } \\
\text { Schulden on ER }\end{array}$ & \\
\hline
\end{tabular}

eine Bank, bei der die Marktteilnehmer ihre Emis-sionsguthaben in Form von Konten unterhalten (vgl. Tabelle 1). Zu Beginn jeder Periode müssen Berichte über geplante Emissionen und deren Deckung durch die Ausstattung bzw. Zuund Verkäufe von Rechten eingereicht werden. Die Behörde prüft dann die globale Verträglichkeit der Pläne mit dem Vermeidungsziel und informiert die Länder. Am Ende der Periode werden dann die endgültigen Berichte erstellt. Da die jährlichen Emissionen nicht perfekt kontrolliert werden können, ist eine erst nachträglich bemerkte Überschreitung der Vorgabe durchaus möglich. 'Banking' und 'borrowing' ermöglichen hier eine flexible Reaktion auf ungeplante Vertragsverletzungen. Beim 'banking' werden Emissionsrechte, die nicht in Anspruch genommen wurden, für zukünftige Perioden angespart, um so eine Flexibilitätsreserve zu schaffen. Beim 'borrowing' nimmt das Land einen Kredit auf zukïnftige Emissionsrechte auf. Eine Überschuldung müßte durch entsprechendes Controlling verhindert werden. Falls auch Unternehmen oder Sektoren am Handel teilnehmen, würden ihre Konten als Unterkonten des nationalen Kontos geführt und mit diesem konsolidiert.

Die Schaffung von Senken (durch Aufforstung etc.) oder die Zerstörung von Treibhausgasen (bei Kohlenwasserstoffen etc.) kann als Erhöhung der Erstausstattung an Emissionsrechten erfaßt werden, ohne das globale Vermeidungsziel zu gefährden. Solange jedoch noch keine zuverlässigen Methoden des Messens und Kontrollierens von Kohlenstoffsenken bestehen, scheidet eine Berücksichtigung aus.

Für den Fall, daß ein Land dauerhaft nicht in der Lage oder Willens ist, seinen Verpflichtungen nachzukommen, müssen Sanktionsmechanismen bestehen. Die Möglichkeiten reichen von negativer Publizität (die vor allem in Demokratien Wirkung entfalten kann) über den temporären Ausschluß vom Handelssystem (was bei Verkäufern von Rechten Anreize zur Vertragseinhaltung induziert) bis zu Sanktionen auf anderen Gebieten.

\section{Systemdynamiken in der Praxis}

Da noch keine Erfahrungen mit einem internationalen Handel mit Emissionsrechten vorliegen, ist nicht zu erwarten, daß von Beginn an ein ideales Marktdesign gelingt, vielmehr dürfte sich das System evolutorisch entwickeln. Ein Vergleich der obigen Skizze mit dem Kioto-Protokoll zeigt, daß man noch weit von der Implementierung selbst eines rudimentären Systems entfernt ist. Weder gibt es derzeit verbindliche jährliche Reduktionsvorgaben als Voraussetzung für Reduktionsgutschriften, noch existiert eine einheitliche nationale Rechnungslegung, die man zur Kontrolle benötigt. Beide Aspekte sollen jedoch bei der nächsten Konferenz in Angriff genommen werden. Aufgrund der wissenschaftlichen Unsicherheit über die Berechnung von Kohlendioxid-Äquivalenten und iber die Klimawirkungen von Senken muß sich zudem in der ersten Phase der Handel auf Kohlendioxid-Emissionen beschränken, eventuell ergänzt um Spezialmärkte für Kohlenwasserstoffe und deren Substitute. Als Handelspartner kommen zunächst nur die westlichen Industrieländer sowie die Transformationsländer in Frage, was Probleme bezuiglich der Marktbreite aufwirft. Da die Industrienationen relativ homogen in ihren Vermeidungskosten sind, wäre Rußland ein Großanbieter.

Schätzungen der Kosteneinsparungen gegenüber einer starren Lösung sind schwierig, da sie von der Ausgestaltung des Systems abhängen. Werte von 50 Prozent werden zwar genannt, sind aber eher als grobe Annäherung zu interpretieren. Die Kosteneinsparungen sind um so höher, je mehr Länder mit unterschiedlichen Vermeidungskosten teilnehmen, je stärker der Handel international und intertemporal organisiert ist und je stärker Innovationen angeregt werden. Gerade in der Anfangsphase dürften die Kosten jedoch relativ hoch sein, weil der Markt noch eng ist, Transaktionen vor allem auf bilateraler Ebene erfolgen und Überwachung und Kontrolle noch wenig standardisiert sind.

Von Bedeutung ist auch, inwieweit nationale 'Mindestvermeidungsklauseln' den Handel beschränken. Zwei Gründe werden für sie angeführt: Bei einem unbeschränkten Handel könnten Entwicklungsländer als Anbieter von Rechten in die Lage kommen, daß diese ihnen nicht mehr zur Verfügung stehen, wenn sie sie selbst benötigen. Daneben würden in Industrieländern bei einem hohen Anteil von zugekauften Reduktionen die nationalen Vermeidungsanstrengungen nicht 
hinreichend angeregt. Eine Lösung dieses Problems bestïnde darin, Emissionsrechte nicht für alle Zeiten zu verkaufen, sondern nur für einen bestimmten Zeitraum zu verpachten. Diese Vorgehensweise ermöglicht den Industrienationen einen langsamen und berechenbaren Ausstieg aus ihrer derzeitigen Technik und senkt so die Kosten des Klimaschutzes.

Das oben angesprochene Problem des 'hot air trading' ist weniger ein Problem des Handels als vielmehr eines der Wahl des Basisjahrs. Natürlich könnte man versuchen, auf Folgekonferenzen realistischere Basisszenarien mit strengeren Vorgaben $\mathrm{zu}$ verhandeln. Ein Erfolg erscheint jedoch zweifelhaft, da weder der derzeitige Hauptemittent USA noch die potentiellen Verkäufer von Emissionsrechten ein Interesse an Nachverhandlungen haben. Es wäre jedoch falsch, durch Mindestvermeidungsklauseln das Verhandlungsergebnis indirekt nachzubessern, da dies die Entwicklung eines Instruments behindert, dessen effizientes Funktionieren die Vereinbarung zukünftiger Protokolle erleichtert.

Auch wenn die Reduktionsziele des Kioto-Protokolls aus wissenschaftlicher Sicht enttäuschend gering sind, können vom Handel mit Emissionsrechten wichtige Impulse für eine Erweiterung der Teilnehmerländer und somit einen effektiveren Klimaschutz ausgehen. Selbst ein sich langsam entwickelnder Handel gibt das Signal, daß die Partizipation am Klimaschutz mit einem Zufluß an Finanzmitteln verbunden sein kann. Anreize zum 'free rider'-Verhalten werden so reduziert.

\section{Anmerkungen}

(1) WBGU: Ziele für den Klimaschutz 1997. Stellungnahme zur dritten Vertragsstaatenkonferenz der Klimarahmenkonvention in Kyoto, Wissenschaftlicher Beirat der Bundesregierung Globale Umweltverönderungen 1997.

(2) Vgl. Wiener, J.B.: Designing Markets for International Greenhouse Gas Control, RFF Climate Issues Brief \#6, Internet Edition, 1997; sowie UNCTAD: Legal Issues Presented by a Pilot International Greenhouse Gas Trading System, UNCTAD/GDS/GFSB/Misc. 1, 1997.

(3) Mullins, F., R.Baron: International GHG Emission Trading, Annex 1 Expert Group on the UN FCCC, Working Paper 9, 1997.

\section{Der Autor}

Or. Wilhelm Althammer ist Prolessor für Makroökonomie an der Handelshochschule Leipzig.

Kontokt: Handelshochschule Leipzig, Jahnallee 59, 04109 leipzig. Tel. 0341/9851-656, Fax: -659.

E-mail: althamm@macroec.hhl.de

Nicht-Regierungs-Organisationen als neue Akteure transnationaler (Umwelt-)Politik

\title{
Substitut des Staatsvolkes?
}

\author{
In der globalisierten Wirtschaft erodieren die klaren Konturen von Staatsge- \\ bief, Staatsvolk und Stautsmacht. Angesichts dieser Entgrenzung gewinnt das \\ Projekt von Global Governance seine Bedeutung. Nicht-Regierungs-Organisa- \\ tionen übernehmen darin eine wichtige Funktion.
}

B Von Elmar Altvater, Achim Brunnengräber und Heike Walk is vor nicht allzu langer Zeit bildeten Nationalstaaten und internationale Institutionen (und Organisationen) den geschlossenen Club der globalen Akteure. Die Welt war in erster Linie Staatenwelt, die sich eine internationale Ordnung gab und „Regime“ zur Regulierung von Handels- und Finanzfragen, zur Bewältigung von Umweltproblemen und zur Abwicklung der Entwicklungshilfe bildeten.

Infolge der Globalisierung aber haben diese traditionellen Akteure eines internationalen politischen Systems gegenüber „den Märkten“ an Einfluß verloren. Die Lösung der vielfältigen Probleme auf der internationalen Ebene wird daher nicht von den nationalstaatlichen Regierungen, aber auch nicht vom freien Spiel der Marktkräfte erwartet. Sie scheint nur noch möglich $\mathrm{zu}$ sein, wenn die Ressourcen der sogenannten ,Zivilgesellschaften“ aller Kontinente mobilisiert werden. Diese neue Konstellation von Akteuren hat auch die ,Commission on Global Governance" vor Augen, wenn sie in ihrem Bericht von 1994 feststellt, daß ,die großen Herausforderungen des folgenden Jahrzehnts nur durch multilateral abgestimmte Vorgehensweisen zu bewältigen seien“ (1) (S.395). Der Bericht der Kommission unterbreitet Handlungsempfehlungen und eröffnet einen Interpretationsspielraum, der bereits dem „ContainerBegriff“ Sustainable Development des Brundtland-Berichtes zur Karriere verhalf: es paßt sehr viel hinein (vgl. Stichwort: Global Governance in diesem Heft). Das Konzept der Global Governance läßt sich allerdings mit demjenigen der zukunftsorientierten Entwicklung systematisch verknüpfen: Die themen- und problemorientierte und die ordnungs- und akteursspezifische Perspektive werden zusammengebracht. So wird die symbolische Integrationskraft für unterschiedliche Handlungs- und Konfliktfelder erzeugt, die notwendig ist, um die
Schnittstelle zwischen der globalen Politik der Nachhaltigkeit und den Akteuren, die für diese Politik verantwortlich zeichnen sollen, zum Funktionieren zu bringen.

Wegen der Vielfalt von Strategien, und weil Global Governance ein politischer Prozeß ist, können die unterschiedlichsten Akteure (aus Politik und Wissenschaft) fast problemlos an das Konzept anknüpfen. Mit dem Konzept der Global Governance wird daher das Terrain für einen Basiskonsens abgesteckt, der es erlaubt, die sozio-ökonomischen Ungleichheiten und ökologischen Krisenszenarien durch die systemische Integration und Kooperation ,alter“ und ,neuer“ Akteure zu lösen. Der Kooperationsansatz soll der Komplexität der Probleme entsprechen, die nicht mehr nur durch die ,invisible hand“ des Marktes und durch die ,visible hand" des Staates bearbeitet werden sollen, sondern in network like forms of governance, also mithilfe der ,third hand“ (Diane Elson) gesellschaftlicher Bewegungen.

Während Politik im Nationalstaat immer und notwendigerweise eine territoriale Dimension hat und Politik Grenzen, gerade territoriale Grenzen setzte, fehlt diese territoriale Dimension im Prozeß der Global Governance. Anstelle der territorialen Verankerung von Politik spielen hier die "Grenzen des Umweltraums" (2) und die Grenzen der sozialen Zumutungen eine beträchtliche Rolle. Sie existieren einerseits ,objektiv“, werden aber im politischen Diskurs von Nicht-Regierungs-Organisationen (engl. Abk: NGOs) und anderen Bewegungen zum Gegenstand der Politik erhoben und von den politischen Institutionen auf allen Ebenen bearbeitet.

\section{NGOS als "nützliche Partner"?}

Allerdings funktioniert die Welt noch nicht nach den Regeln von Global Governance. Denn die westlichen Regierungen diktieren weitgehend die Funktionsweisen und Bedingungen der globalen Deregulation durch Weltbank, Internationalen 
(c) 20I0 Authors; licensee IÖW and oekom verlag. This is an article distributed under the terms of the Creative Commons Attribution Non-Commercial No Derivates License (http://creativecommons.org/licenses/by-nc-nd/3.o/), which permits unrestricted use, distribution, and reproduction in any medium, provided the original work is properly cited. 